\title{
Effects of aryl-hydrocarbon ligands on dendritic cell maturation
}

\author{
Hana'a A. Abu-Rezq ${ }^{1,}$, , Douglas G. Millar ${ }^{2}$ \\ ${ }^{1}$ Aridland Agricultural Production Program, Environment and Life Sciences Centre, Kuwait Institute for Scientific Research, Shuwaikh, \\ Kuwait \\ ${ }^{2}$ The University of Manchester, Faculty of Life Sciences, Immunology Group, Manchester, United Kingdom
}

Email address:

haborizq@kisr.edu.kw (H. A. Abu-Rezq), Douglas.millar@manchester.ac.uk (D. G. Millar)

To cite this article:

Hana'a A. Abu-Rezq, Douglas G. Millar. Effects of Aryl-Hydrocarbon Ligands on Dendritic Cell Maturation. International Journal of Immunology. Vol. 1, No. 3, 2013, pp. 24-34. doi: 10.11648/j.iji.20130103.11

\begin{abstract}
Aryl-hydrocarbon receptor (AhR) is a cytosolic receptor found in many cells, including immune cells, and its function has been implicated in metabolic and transcriptional control of immune regulation. In the present study we have investigated the effect of the AhR-ligands, FICZ, I3C, curcumin, quercetin and the ligands precursor tryptophan, on bone marrow-derived dendritic cell (BMDC) maturation and immuno-stimulatory or immuno-suppressive phenotypes. We find that immature and mature BMDC express intracellular AhR. Treatment of BMDC with AhR-ligands during LPS-induced BMDC maturation had no significant effect on the expression of MHC class II, CD40 and CD86, with the exception of I3C which suppressed CD40 expression by BMDC at high doses. However, all AhR-ligands significantly enhanced the secretion of pro-inflammatory cytokines, including IL-6, IL-12p40, TNF- $\alpha$, and IL- $1 \beta$. In contrast, only the AhR-ligands FICZ and I3C increased IL-10 and TGF- $\beta$ secretion. Tryptophan, curcumin, and quercetin significantly suppressed IL-10 secretion without affecting TGF- $\beta$ secretion. Finally, FICZ and I3C significantly enhanced the expression of the tolerogenic DC enzyme, indoleamine-2, 3-dioxygenase (IDO), while tryptophan, curcumin and quercetin did not change IDO expression. These results suggest that FICZ and I3C can promote a tolerogenic BMDC phenotype consistent with suppression of immune responses by enhancing the secretion of anti-inflammatory cytokines and increasing IDO expression. In contrast, tryptophan, curcumin and quercetin can promote an immuno-stimulatory BMDC phenotype, secreting elevated pro-inflammatory cytokines, which could help in skewing T cell responses towards the development of effector CD4 and CD8 T cell subsets.
\end{abstract}

Keywords: Curcumin, Tryptophan, Quercetin, FICZ, I3C, TGF- $\beta$, IL-10, IDO

\section{Introduction}

The aryl-hydrocarbon receptor (AhR) is a cytosolic sensor found in several immune cell types. These include $\mathrm{T}$ cell subsets such as IL-17-producing T helper cells (Th17), regulatory $\mathrm{T}$ cells (Treg), and $\gamma \delta$-T cells $[1,2]$, as well as macrophages and dendritic cells (DC) $[3,4]$. The AhR can recognize different compounds including environmental toxins, natural dietary compounds, and endogenous tryptophan-derived metabolites [5]. Once these ligands bind to the receptor, nuclear translocation occurs and the receptor binds to AhR nuclear translocator (ARNT) and the AhR:ARNT complex binds to a specific sequence on DNA called the dioxin response element (DRE), and this triggers transcription of genes including cypla1, encoding the detoxifying enzyme cytochrome P450 [5,6]. Since AhR is present in innate immune cells and can recognize environmental compounds, this raises the possibility that the AhR may act as a pattern recognition receptor (PRR) for metabolic or nutritional status and for induction of different immune responses.

DCs are thought to regulate the stimulation of immunity versus maintenance of peripheral tolerance by several mechanisms [7]. T cell deletion or anergy can result from antigen presentation by steady-state immature DC in the absence of co-stimulatory signals. Induction of Treg cells can also occur following antigen presentation in the presence of TGF- $\beta$, leading to long-lasting tolerance [8]. Pathogen detection by DC via PRR can signal for pro-inflammatory cytokine production and co-stimulatory molecule expression, which promote polarized helper $\mathrm{T}$ cell responses (Th1, Th2, Th17) and abrogate tolerance induction. A pro-inflammatory environment rich in IL-12 results in Th1 polarization, whereas IL-4 results in Th2 cells, 
and TGF- $\beta$ with either IL- 6 , IL-1 $\beta$ or IL-21 results in Th17 effector cells $[9,10]$.

However, environmental conditioning of DC can increase tolerogenic phenotypes and function as well. Increased IL-10 production is thought to skew to a type 1 regulatory T cell $(\operatorname{Tr} 1)$ phenotype [11]. Retinoic acid appears to promote conversion of intestinal DC to a tolerogenic / regulatory phenotype [12]. Exposure of DC to IFN- $\gamma$ is also known to confer a tolerogenic phenotype by the induction of indoleamine 2, 3 dioxygenase (IDO) expression [13]. IDO is an enzyme that breaks down the amino acid tryptophan, increasing the production of its metabolite kynurenine. IDO expression limits $\mathrm{T}$ cell activation at the site of inflammation by consuming essential tryptophan and increases the development of Treg, possibly via AhR-dependent kynurenine activity $[14,15]$. These observations illustrate how the stimulating conditions can affect cytokine production, regulatory enzyme expression, and function of DC. Identification of pattern recognition pathways that promote tolerogenic DC function could have therapeutic potential for treatment of autoimmune conditions.

Previously, the AhR-ligands 3, 7, 8-tetrachlorodibenzo$p$-dioxin (TCDD) and 6-formylindolo $[3,2-b]$ carbazole (FICZ) were reported to induce IDO gene expression in monotypic cell line-derived DC [16] and in bone marrow-derived DC (BMDC) [17, 18]. Interestingly these compounds are also reported to promote Treg and Tr1 cell development via an AhR-dependent mechanism in $\mathrm{T}$ cells $[19,20]$. In vivo, TCDD/dioxin is known to produce immuno-suppression and a recent study reported that oral administration of TCDD reduced the population of splenic CD11 $\mathrm{c}^{\text {high }}$ DC [21]. However this effect was associated with increased CCR7 expression and elevated CD86, CD54, CD40, and MHC class II positive activated DC phenotype in lymph nodes, suggesting altered homeostasis of DC, rather than a direct cytotoxic or suppressive effect on DC. Long term exposure to TCDD or FICZ, in vitro, also increased the activated, mature DC phenotype, following LPS treatment [21]. These results suggest that the effects of AhR activation on DC might not be exclusively immuno-suppressive.

In fact, other studies, have demonstrated that FICZ can promote AhR-dependent Th17 $\mathrm{T}$ cell differentiation and worsen autoimmune disease severity in a model of multiple sclerosis [22, 23]. Furthermore, natural AhR-ligands derived from aromatic amino acids increase Th17 development, in vitro [24]. Therefore, it appears that a combination of potential effects of different AhR-ligands on DC might confer either an immuno-suppressive or mature, pro-inflammatory phenotype, and this raises the possibility that different responses to AhR ligation might result in either tolerogenic or immunogenic adaptive immune responses.

In the present study, we have investigated the effect of different AhR-ligands including; FICZ, I3C, curcumin, quercetin, or ligand precursor tryptophan, on BMDC viability, maturation, pro-inflammatory cytokine secretion, anti-inflammatory cytokine secretion and the expression of intracellular IDO. Our results show that none of the
AhR-ligands tested have a significant effect on LPS-induced BMDC surface maturation markers, except for the suppressive effect of I3C on CD40 expression by BMDC at high doses. However, all AhR-ligands enhanced the LPS-induced secretion of pro-inflammatory cytokines from BMDC. In contrast, only FICZ and I3C significantly increased anti-inflammatory cytokines while tryptophan, curcumin and quercetin significantly suppressed IL-10 and had no effect on TGF- $\beta$ secretion by BMDC. Furthermore, we confirm the ability of FICZ to enhance the expression of IDO in BMDC, and find that $\mathrm{I} 3 \mathrm{C}$ has a similar ability, but that other ligands or the ligand precursor tryptophan are not able to stimulate IDO expression. These results suggest that FICZ and I3C can promote a tolerogenic BMDC phenotype consistent with the development of Treg and $\operatorname{Tr} 1$ polarization. In contrast, tryptophan, curcumin and quercetin can promote a more immuno-stimulatory BMDC phenotype that might enhance the development of effector $\mathrm{T}$ cell subsets.

\section{Materials and Methods}

\subsection{Chemical and Antibodies}

6-formylindolo [3, 2-b] carbazole (FICZ) was purchased from Enzo Life Science (UK). Indole-3-carbinol (I3C), curcumin (was purchased from sigma at the highest purity available $>94 \%$ ), quercetin, tryptophan, formalin solution neutral buffered (NBF), tween-20, $\beta$-mercapto-ethanol, nigericin, and LPS (E.coli serotype 026:B6) were purchased from Sigma (Poole UK). All antibodies used for ELISA and all anti-cytokine and BMDC surface marker antibodies used for FACS analysis were from eBioscience. Avidin-HRP, permeabilization and fixation buffer were purchased from eBioscience (UK). Tetra-methyl-benzidine (TMB) was purchased from Alpha Labs. WST-1 reagent used for viability test was from Roche (UK). Saponin was purchased from Fluka (Switzerland). RPMI, HBSS, L-glutamine, penicillin, streptomycin, fetal bovine serum and Alexa-Fluor448 goat anti-Rabbit IgG were purchased from Invitrogen (UK). Rabbit anti-AhR antibody was obtained from Aviva System biology. Rat monoclonal IDO antibody was purchased from Novus Biological (UK).

\subsection{Murine BMDC Preparation}

BMDC were prepared from $\mathrm{C} 57 \mathrm{Bl} / 6 \mathrm{~J}$ mice as previously described [25]. Briefly, C57Bl/6J mice were sacrificed, hind legs were dislocated at the pelvic joint, skin and muscles around legs and fibula bone were discarded. Bones were cut at both ends and the marrow cells flushed out using a syringe and 25 gauge needles, letting the media flow into a sterile Petri dish, and same needle was used to break up cell aggregates. Cells were then filtered through $100 \mu \mathrm{m}$ nylon mesh, centrifuged for $10 \mathrm{~min}$ at $1500 \mathrm{rpm}$, supernatant was discarded, ACK buffer $\left(0.15 \mathrm{M} \mathrm{NH}_{4} \mathrm{Cl}, 10 \mathrm{mM} \mathrm{KHCO}_{3}\right.$, $0.1 \mathrm{mM}$ EDTA) was added for $2 \mathrm{~min}$ to lyses red blood cells. Cells were then washed by adding HBSS buffer, cells were 
centrifuged, supernatants were discarded, and cells were re-suspended in fresh media (RPMI $+10 \%$ heat-inactivated-FBS $+\mathrm{L}$-glutamine + Pen $/$ Strep $+50 \mu \mathrm{M}$

BME). Cells were counted and the number was adjusted to $2 \times 10^{6}$ cells $/ 10 \mathrm{ml}$. Cells were cultured in sterile Petri dishes and incubated for 6 days at $37^{\circ} \mathrm{C}, 5 \% \mathrm{CO}_{2}, 95 \%$ humidity and on day three cells were supplemented with cRPMI and 40ng/ml GM-CSF (GM-CSF obtained from conditioned media from Ag8.653 cell line secreting GM-CSF) (Zal et al., 1994).

\subsection{BMDC Maturation with LPS and Detection of Intra-Cellular AhR}

BMDC were harvested on day 6 , recovered by centrifugation at $1500 \mathrm{rpm}$ for $5 \mathrm{~min}$, re-suspended in fresh complete RPMI media and the cell concentration was adjusted to $2 \times 10^{6}$ cells per $\mathrm{ml}$. $1 \mathrm{ml}$ of BMDC were transferred to a 24-well tissue culture plate and $200 \mathrm{ng} / \mathrm{ml}$ LPS was added to some wells. Plates were incubated overnight at $37^{\circ} \mathrm{C}, 5 \% \mathrm{CO}_{2}, 95 \%$ humidity. The following day, cells were washed with FACS buffer (PBS, 2\% heat inactivated-FBS, $0.1 \% \mathrm{NaN}_{3}$ ), and cells were stained with

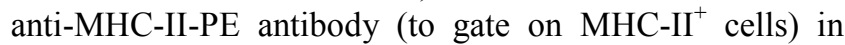
FACS buffer for $30 \mathrm{~min}$ at $4^{\circ} \mathrm{C}$. Maturation was confirmed by staining some samples with; anti-CD11c-PE, anti-CD40-PE or MHC-II-PE or anti-CD86-PE. Cells were washed with FACS buffer, fixed and permeabilized with Fix-Perm solution (NBF, $0.5 \%$ heat inactivated-FBS, $0.1 \%$ Saponin) for $30 \mathrm{~min}$ at room temperature. After incubation time, cells were washed with Perm-Wash buffer (FACS buffer, 0.1\% Saponin), and then cells were stained with rabbit polyclonal anti-AhR antibody (Aviva) in Perm-Wash buffer and incubated for $30 \mathrm{~min}$ at room temperature. Cells were washed with Perm-Wash buffer, and BMDC were stained with secondary AlexaFluor488-conjugated goat anti-Rabbit IgG antibody in Perm-Wash buffer and cells were incubated at room temperature for $30 \mathrm{~min}$ (all antibodies were used at 1:1000 dilutions of $1 \mathrm{mg} / \mathrm{ml}$ ). Cells were washed and re-suspended in isotonic solution and fluorescence data acquired using FACS-Calibur Flow Cytometer (BD Bioscience) using CellQuest software and the obtained data were analyzed by FlowJo software (Tree Star, USA).

\subsection{Cytotoxicity Assay}

BMDC were harvested on day 6 , adjusted to $1 \times 10^{6}$ cells per $\mathrm{ml}$ and were transferred to a 96 -well tissue culture plate. Cells were exposed to 1:3 serial dilutions of different AhR-ligands (as indicated in the figure legend), HBSS buffer alone (positive control for viability), or 5\% DMSO (negative control for viability), in triplicate and incubated overnight at $37^{\circ} \mathrm{C}, 5 \% \mathrm{CO}_{2}, 95 \%$ humidity. To the cells, $1: 2$ diluted WST-1 reagents in HBSS buffer was added to all wells, mixed gently by tapping and the plates were incubated at $37^{\circ} \mathrm{C}, 5 \% \mathrm{CO}_{2}, 95 \%$ humidity for $8 \mathrm{hrs}$. Absorbance at $450 \mathrm{~nm}$ for WST-1 was measured using a DYNEX Plate Reader and Revelation Software, and the values used to calculate \% viability, compared to the viable HBSS positive control.

\subsection{BMDC Maturation with LPS and AhR-Ligands}

BMDC were harvested on day 6 , adjusted to $2 \times 10^{6}$ cells per $\mathrm{ml}$ and $2 \times 10^{5}$ cells were transferred to each well of a 96-well tissue culture plate. Different concentrations of AhR-ligands were added to the appropriate wells with and without $200 \mathrm{ng} / \mathrm{ml}$ LPS. Plates were incubated overnight at $37^{\circ} \mathrm{C}, 5 \% \mathrm{CO}_{2}, 95 \%$ humidity and then centrifuged for $5 \mathrm{~min}$ at $1500 \mathrm{rpm}$, supernatants were collected and stored at $-80 \mathrm{oC}$ for cytokine determination by ELISA, and cell pellets were stained with the following markers of DC and maturation: anti-CD11c-PE, anti-MHC-class-II-PE, anti-CD40-PE, anti-CD86-PE (all antibodies were used at 1:1000 dilutions of $1 \mathrm{mg} / \mathrm{ml}$ ). The plate was incubated at $4^{\circ} \mathrm{C}$ for one hour, then it was centrifuged, supernatants were discarded, cells were fixed in NBF, and fluorescence data acquired using a FACS-Calibur flow cytometer (BD Bioscience) using CellQuest Software and analyzed using FlowJo software (Tree Star, USA).

\subsection{BMDC Cytokine Secretion Following Treatment with LPS and AhR-Ligands}

BMDC $\left(3 \times 10^{4}\right.$ cells per well) were treated in 96-well plates with the following compounds: 200ng/ml LPS

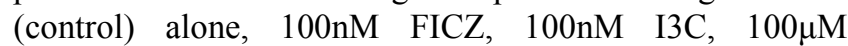
tryptophan, $10 \mu \mathrm{M}$ curcumin, $10 \mu \mathrm{M}$ quercetin, and $200 \mathrm{ng} / \mathrm{ml}$ LPS was added to all wells containing AhR-ligands. Plates were incubated overnight at $37^{\circ} \mathrm{C}, 5 \% \mathrm{CO}_{2}, 95 \%$ humidity. Next day, $2 \mu \mathrm{M}$ nigericin was added to some samples of BMDC for $8 \mathrm{hrs}$ to promote processing and secretion of IL-1 $\beta$. BMDC were centrifuged at $1500 \mathrm{rpm}$ for $5 \mathrm{~min}$, supernatants were collected and stored at $-80^{\circ} \mathrm{C}$ until measurement of IL-12p40, IL-6, TNF- $\alpha$, IL-1 $\beta$, IL-10 and TGF- $\beta$ by ELISA, (for TGF- $\beta$ ELISA, samples were not acidify, thus the TGF- $\beta$ measured was the naturally occurring free TGF- $\beta$ ).

\subsection{Enzyme-Linked Immunosorbent Assay (ELISA)}

ELISA plates (NUNC Immuno Maxisorp) were coated with purified capture antibody in the recommended coating buffer (100ng/well) and blocked with ELISA blocking buffer (PBS $+2 \%$ BSA). Serial dilutions of recombinant mouse cytokines were added to the plates to prepare the standard curve. Samples were transferred to the rest of the wells, and incubated for two hours at room temperature. After incubation and wash, biotin-conjugated anti-cytokine detection antibody (used at 1:1000 dilutions) and avidinHRP (1:2000) were added. After a final wash, TMB substrate solution was added to all wells and absorbance at $630 \mathrm{~nm}$ was measured using Plate Reader and Revelation Software. 


\subsection{BMDC Maturation with LPS for Detection of Intra-Cellular IDO}

BMDC $\left(3 \times 10^{4}\right.$ cells per well) were treated in 96-well plates with and without $200 \mathrm{ng} / \mathrm{ml}$ LPS, and different AhR-ligands were added to some wells. Cells were incubated overnight at $37^{\circ} \mathrm{C}, 5 \% \mathrm{CO}_{2}, 95 \%$ humidity. The following day, cells were washed with FACS buffer, and then stained with anti-CD11c-biotin antibody in FACS buffer for $30 \mathrm{~min}$ at $4^{\circ} \mathrm{C}$. Cells were washed with FACS buffer, fixed and permeabilized with foxp3 fixation/permeabilization solution (eBioscience) for $30 \mathrm{~min}$ at room temperature. After incubation, cells were washed with Perm-Wash buffer, stained with rat monoclonal anti-IDO antibody (Novus) in Perm-Wash buffer and incubated for $30 \mathrm{~min}$ at room temperature. Cells were washed with Perm-Wash buffer, and BMDC were stained with anti-rat antibody PE-conjugated (eBioscience) for another 30min in Perm-Wash buffer (all antibodies were used at 1:1000 dilutions of $1 \mathrm{mg} / \mathrm{ml}$ ). Cells were washed and re-suspended in NBF and fluorescence data acquired using a FACS-Array flow cytometer (BD Bioscience) and data were analyzed using FlowJo Software.

\subsection{Statistics}

Significant differences between experimental groups were determined using ANOVA or Student's $t$ test by using GraphPad Prism 4 software and all bars show SEM.

\section{Results}

\subsection{Mature and Immature BMDC Express Intracellular AhR}

To investigate whether immature and mature BMDC express the intracellular AhR or not, BMDC were treated with or without LPS and then stained with anti-AhR antibody and cells were examined by flow cytometry. Immature and mature BMDC both displayed detectable intracellular AhR staining, compared to isotype control antibody (Fig. 1A \& B). Maturation did not significantly alter the level of AhR expression (Fig. 1C). The LPS-matured BMDC were characterized by higher expression of maturation markers MHC-II, CD40, and CD86 on their surfaces.

\subsection{High Doses of Curcumin and Quercetin Reduce the Viability of BMDC}

Next, the effect of AhR-ligands and precursor compounds on BMDC viability was tested. BMDC were treated with doses of the AhR-ligands FICZ, I3C, tryptophan, curcumin, and quercetin and cells were incubated overnight. Doses were chosen to cover ranges of FICZ previously reported to affect $\mathrm{T}$ cell polarization, in vitro $[22,23]$, I3C up to doses previously found to reduce spleen cell viability (Abu-Rezq $\&$ Millar, unpublished data), curcumin at doses previously reported to induced AhR-dependent EROD activity [26], quercetin at doses previously reported to stimulate EROD activity [27], and tryptophan doses previously reported to have effects on T cell responses [24]. The following day, cell viability was measured. FICZ had no significant effect on BMDC viability up to $100 \mathrm{nM}$ as compared to BMDC treated with buffer alone (Fig. 2A). Similarly, I3C had no significant effect on BMDC viability up to 100nM (Fig. 2B). Also, tryptophan, a normal component of RPMI media at $24 \mu \mathrm{M}$, had no significant effect on BMDC viability up to $1 \mathrm{mM}$ (Fig. $2 \mathrm{C})$. In contrast, curcumin at doses greater than $10 \mu \mathrm{M}$ caused a significant reduction in BMDC viability (Fig. 2D). Similarly, quercetin at doses of $30 \mu \mathrm{M}$ or more caused a significant decrease in BMDC viability (Fig. 2E). Since FICZ and I3C condensation products such as a DIM or ICZ, are reported agonists of the AhR in the nano- to micro-molar range [22, 28], these results confirm the range of appropriate doses to use on BMDC. However, these results indicate that curcumin and quercetin can exert cytotoxic effects on BMDC at doses exceeding 10-30 $\mu \mathrm{M}$.

\subsection{AhR-Ligands do not Alter LPS-Induced Surface Maturation of BMDC}

The effect of AhR-ligands on BMDC maturation was also examined. BMDC were treated with or without LPS and stimulated with doses of different AhR-ligands or precursor compounds, and then cells were stained for surface maturation markers. LPS-treatment of BMDC resulted in up-regulation of the surface markers MHC class II, CD40, and CD86 (Fig. 3). Treating BMDC with LPS and different AhR-ligands had no significant effect on the expression of MHC class II, CD40, or CD86 as compared to BMDC treated with LPS alone (Fig. 3A-C), except the suppressive effect of I3C on CD40 expression by BMDC at high doses (Fig. B). Therefore, at doses that are non-toxic to BMDC, none of the AhR-ligands or precursor compounds tested significantly diminished or enhanced the ability of LPS to mature the BMDC (the effect of AhR-ligands on the expression of specific maturation markers after treating BMDC with sub-optimal doses of LPS was not tested, but none of the AhR-ligands alone have shown any significant effect on the expression of maturation markers by immature BMDC.

\subsection{AhR-Ligands Increase Pro-Inflammatory Cytokine Secretion by BMDC}

To investigate whether AhR-ligands affect secretion of pro-inflammatory cytokines by BMDC, cells were treated with and without LPS and FICZ, I3C, tryptophan, curcumin and quercetin. The following day, supernatants were collected and the amount of IL-6, IL-12p40, TNF- $\alpha$ and IL-1 $\beta$ were measured by ELISA. Nigericin, a potassium ionophore, was added to some samples in order to promote IL-1 $\beta$ processing and secretion. BMDC treated with LPS alone showed increased secretion of all the pro-inflammatory cytokines (Fig. 4). Additional treatment with FICZ, I3C, tryptophan, curcumin or quercetin caused a 
significant $(* * p<0.01)$ increase in LPS-induced IL-6 secretion from BMDC, compared to LPS alone (Fig. 4A). Similarly, BMDC stimulated with LPS and any of the AhR-ligands showed significantly $(* * p<0.01)$ increased IL-12p40 secretion (Fig. 4B). Also, BMDC treated with LPS and any of the AhR-ligands showed significantly $(* * p<0.01)$ increased TNF- $\alpha$ secretion (Fig. 4C). Treatment with LPS and all AhR-ligands also significantly $(* p<0.05)$ increased the priming of BMDC to secrete IL-1 $\beta$, as compared to treatment with LPS alone (Fig. 4D). Interestingly, these effects of FICZ and I3C on IL-6, IL-12p40 and IL- $1 \beta$ responses, but not TNF- $\alpha$ secretion, were significantly $(* p<0.05)$ lower than tryptophan, curcumin, or quercetin, suggesting that these AhR-ligands may have less pro-inflammatory activity on BMDC. These results suggest that all the AhR-ligands or precursor compounds promote increased pro-inflammatory activity by LPS-activated BMDC.

\subsection{FICZ and I3C Increase Anti-Inflammatory Cytokine Secretion by BMDC}

The effect of AhR-ligands on anti-inflammatory cytokines was also measured. LPS-treatment induced detectable secretion of both IL-10 and TGF- $\beta$ from BMDC (Fig. 5). FICZ or I3C treatment together with LPS significantly $(* * p<0.01)$ increased IL-10 secretion by BMDC compared to LPS alone (Fig. 5A). In contrast, including tryptophan, curcumin, or quercetin with LPS caused a significant $(* * p<0.01)$ suppression of IL-10 secretion (Fig. 5A). Similarly, BMDC treated with LPS and FICZ or I3C displayed a significant $(* * p<0.01)$ increase in TGF- $\beta$ secretion (Fig. 5B). Inclusion of tryptophan, curcumin, or quercetin with LPS did not have any effect on TGF- $\beta$ secretion (Fig. 5B). The effects of AhR-ligand exposure on LPS-treated retinoic acid (RA)-imprinted BMDC, were also examined and showed similar results to those of conventionally prepared BMDC.

\subsection{FICZ and I3C Enhance Intra-Cellular Expression of IDO in BMDC}

The effect of the AhR-ligands on IDO expression was then examined. BMDC pellets from samples treated as in the previous experiments were stained for CD11c and IDO. LPS-treated-BMDC significantly increased the expression of IDO as compared with immature BMDC (minus LPS), $(* * p<0.01)$ (Fig. 6 A\&B). BMDC treated with LPS and either FICZ or I3C showed a significant $(* * * p<0.001)$ increase in IDO expression compared to LPS alone (Fig. 6 A\&B). In contrast, treatment with LPS and either tryptophan, curcumin, or quercetin caused no change in IDO expression compared to BMDC-treated with LPS only (Fig. 6 A\&B). Together, these results demonstrate that FICZ and $\mathrm{I} 3 \mathrm{C}$ have distinct effects on BMDC that enhance an immunosuppressive phenotype, whereas tryptophan, curcumin, and quercetin, at non-toxic doses, promote a more immuno-stimulatory phenotype with augmented production of pro-inflammatory cytokine and reduced production of anti-inflammatory cytokines.

\section{Discussion}

We have demonstrated for the first time that different AhR-ligands or ligand precursors can either increase the immuno-stimulatory phenotype of BMDC, or increase an anti-inflammatory and tolerogenic phenotype. The effects of different ligands emphasize the potential activity of the AhR in innate immune cells that might result in suppressed immune responses and be beneficial in therapy of auto-immune disease, or alternatively could increase effector responses and therefore benefit vaccination.

In vivo, TCDD is known to result in suppression of $\mathrm{T}$ cell-mediated immune responses [29]. Investigations into the effects of TCDD and other AhR-ligands on DC phenotype and function have produced conflicting results. Previous studies examined the effects of the AhR-ligands TCDD, FICZ, and 2- (1'H-indole-3'- carbonyl)-thiazole4-carboxylic acid methyl ester (ITE) on DC differentiation and function. Treatment of BMDC with either vehicle alone or with TCDD, FICZ or ITE for 7 days, showed that all the AhR-ligands suppressed the expression of CD11c but increased MHC class II, CD86 and CD25 on the BMDC [17]. After LPS-treatment, TCDD-exposed BMDC showed a greater increase in $\mathrm{MHC}$ class II expression and pro-inflammatory cytokine secretion. The effects of TCDD were dependent on AhR-mediated gene transcription. However, the TCDD-exposed BMDC also showed up-regulation of markers associated with a regulatory phenotype, including IDO and TGF- $\beta 3$. In a separate study from the same group, administration of TCDD by gavage to mice, showed a decreased frequency and number of splenic CD11chigh DC, compared to the control [21]. TCDD administration increased CD86 and CD54, but decreased the expression of CD11a and MHC class II on CD11chigh DC. However, it was observed that after TCDD administration, splenic DC expressed higher levels of CCR7, indicating a migratory phenotype, and $\mathrm{CD} 11 \mathrm{c}^{+}$cells in the lymph node displayed increased MHC class II and CD40, suggesting maturation. Additional studies again demonstrated mixed effects of TCDD, FICZ and ITE on the phenotype of Flt3L-generated BMDC, in vitro [18]. All three ligands were reported to increase expression of surface maturation markers, including MHC Class II, CD86, and CD54. Following strong activation with TLR-ligands, however, TCDD suppressed the secretion of both pro- and anti-inflammatory cytokines from the BMDC.

These reports differ significantly from our study in several important respects. First, we used immature DC on day 6 of development in GM-CSF $(75-80 \%$ of DC are CD11 $\mathrm{c}^{+}$cells), which expressed low MHC class II, CD40, and CD86, and saw no change in these markers on DC after overnight treatment with AhR-ligands or precursor compounds alone. Our BMDC, developed in GM-CSF, showed strong up-regulation of MHC class II, CD40 and 
CD86 following LPS treatment, however, none of the AhR-ligands tested had significant effects on the extent of up-regulation. Second, our BMDC were generated in the absence of any AhR-ligand, and only exposed to AhR-ligand for up to $24 \mathrm{hrs}$ during overnight stimulation with or without LPS. However, similar to the effects of TCDD-exposed GM-CSF-generated BMDC on cytokine secretion following LPS-treatment, reported by Bankoti et al. [17], we observed increased IL- 6 and TNF- $\alpha$ secretion by GM-CSF-generated BMDC induced by overnight treatment with all the compounds we tested. Our findings suggest that acute exposure to a variety of AhR-ligands, is associated with mixed pro- and anti-inflammatory cytokine response, not always associated with "tolerogenic" DC markers.

The ability of DC to promote tolerance requires acquisition of a tolerogenic phenotype [7, 30]. This can include expression of IDO, ILT3/4, PD-L1, Fas, IL-10, and/or TGF- $\beta$. TCDD and FICZ were previously reported to increase IDO expression in DC [16-18]. Interestingly, the IDO breakdown product of tryptophan, kynurenine, has been found to promote Treg in an AhR-dependent manner [14]. Vogel et al. [16] showed that Foxp $3^{+}$Treg increased following TCDD administration to mice, and this increase was blocked by 1-methyl-tryptophan, a competitive inhibitor of IDO. Together, these findings suggest that some AhR-ligands may promote a tolerogenic environment by both directly increasing Treg differentiation, and by indirectly increasing additional Treg-promoting AhRligands via IDO induction.

Another tolerogenic phenotype is characterized by IL-10 production from DC, influencing Tr1 cell development [11]. In addition to the reported effects of some AhR-ligands on Treg skewing, TCDD and FICZ were reported to promote Tr1 cell differentiation via transcriptional activation pathways directly affecting the IL-10 promoter [19, 20]. Similar activation of IL-10 production in DC may also contribute to a tolerogenic cytokine environment induced by some AhR-ligands. As we have demonstrated, however, only FICZ and I3C seem to be capable of inducing IL-10 secretion from BMDC, while the other natural AhR-ligands or elevated tryptophan suppressed LPS-induced IL-10 responses (Fig. 5). These differential abilities of AhR-ligands or precursor compounds to induce IL-15, TGF- $\beta$ and IDO in BMDC suggest that these compounds may have distinct effects on DC-induced T cell responses.

Accumulating evidence has shown that other natural AhR-ligands, such as curcumin, have anti-inflammatory, anti-oxidant, and anti-carcinogenic activities [31]. A previous study examined the effects of curcumin on immunogenic or tolerogenic BMDC phenotypes [32]. Results showed that treatment of BMDC with $50 \mu \mathrm{M}$ curcumin, enhanced the expression of IL-10 but reduced expression of inflammatory cytokines including TNF- $\alpha$ and IL-6. Furthermore, BMDC treatment with curcumin enhanced the development of $\mathrm{CD} 25^{+}$Foxp $3^{+}$Treg and IL-10-producing Tr1 cells, and consequently suppresses colitis, in vivo. These results are in contrast with the enhanced pro-inflammatory effects and reduced antiinflammatory effects of curcumin seen in our study (Fig. 4 \& 5). One possible explanation for this difference is the potentially cytotoxic dose of curcumin used in the previous study $(50 \mu \mathrm{M})$, which we found would cause greater than $50 \%$ reduction in viability of BMDC (Fig. 2). Therefore, the suppressive effect on colitis may be due to a high level of DC apoptosis following a high dose of curcumin treatment, and the resulting up take of the apoptotic cells by the remaining live DC, which could enhance the secretion of IL-10.

Another previous study examined whether curcumin could influence surface molecule expression, cytokine production, and their signaling pathways in BMDC [33]. Results showed that curcumin significantly decreased the expression of CD80, CD86, and MHC class II on BMDC. In addition, the expression of IL-12 and secretion of pro-inflammatory cytokines including IL-1 $\beta$, IL-6, and TNF- $\alpha$ were suppressed by curcumin (curcumin was purchased from Sigma at the highest purity available $>94 \%$ ). We presently cannot explain why our data conflict with this previous study, except for the possibility that the responses of BMDC were generated with GM-CSF and IL-4, differ from those used in our study, generated with GM-CSF alone.

Another reported property of curcumin, is its ability to inhibit IFN- $\gamma$-induced IDO expression [34, 35]. While suppression of IDO correlated with a reduction in co-stimulatory molecule expression, the effect of curcumin was also accompanied by restoration of $\mathrm{T}$ cell responses. These results suggest that down-regulation of IDO in BMDC by curcumin may be able to increase immune responses, and reverse immuno-suppressive mechanisms. Immuno-suppressive effects of quercetin on the activation and function of murine BMDC have also been reported [36]. Quercetin, in the dose range of $6-50 \mu \mathrm{M}$, reduced the expression of pro-inflammatory cytokines, chemokines, MHC class II and co-stimulatory molecules. Furthermore, Ag-specific T cell activation, both in vitro and in vivo, was inhibited after treating BMDC with quercetin. Overall, the inflammatory responses of the BMDC used in this study were low and the cells were used at a later stage of development (day 8-12), compared to our results which found no effect of quercetin on immature (day 6) BMDC surface maturation and increased levels of pro-inflammatory cytokine secretion (Fig. $3 \& 4$ ).

The effects we observed with tryptophan addition to BMDC cultures, increasing pro-inflammatory cytokine secretion, is consistent with the reported ability of natural AhR-ligands derived from aromatic amino acids in culture media to enhance Th17 cell differentiation in vitro [24]. While these compounds may directly activate AhR in T cells during Th17 differentiation, we have also noted that culture media rich in aromatic amino acids (eg. IMDM), enhances BMDC maturation [37], suggesting that natural AhR-ligands may also promote an immuno-stimulatory DC phenotype.

How might different AhR-ligands have either mixed 
inflammatory and regulatory effects on BMDC, while others produce exclusively pro-inflammatory responses? One possibility is that different affinity AhR-ligands induces distinct gene expression profiles [38]. The transcriptional signature of DC from mice exposed to TCDD shows down-regulation of many genes, most known to contain a DRE [4]. The authors proposed that endogenous antagonists generated in different tissues might affect the gene expression profiles. Expression profiling of DC exposed to other AhR-ligands, has not been reported. However, curcumin is known to be turned over more slowly ( $>48 \mathrm{hrs}$ ) [26], and can act in an antagonistic manner on AhR-ligand breakdown by cytochrome P450 [39]. Therefore, short-lived or sustained AhR activation, coupled with distinct agonist/antagonist effects might explain how different ligands can induce various responses. It will be interesting to examine whether the AhR-ligands which enhance a tolerogenic DC phenotype, will antagonize the activity of other natural AhR-ligands, or vice versa. As most dietary AhR-ligands have affinities lower than artificial environmental contaminants, but can both antagonize AhR-activation by TCDD and induce DRE-regulated gene activation in the physiological micro molar range $[5,26 \&$ 40], effects of ligand combinations may be complex.

In conclusion, our results demonstrate that different AhR-ligands or precursor compounds have distinct effects on BMDC, associated with immuno-suppressive or immuno-stimulatory responses. Ligands that induce a tolerogenic DC phenotype (FICZ, I3C) have the potential to be used in therapy of autoimmune disease or transplantation tolerance. Ligands that induce an immunogenic DC phenotype may worsen inflammation and autoimmunity, but have the potential to enhance cell-based anti-cancer vaccination.

\section{Results}

A
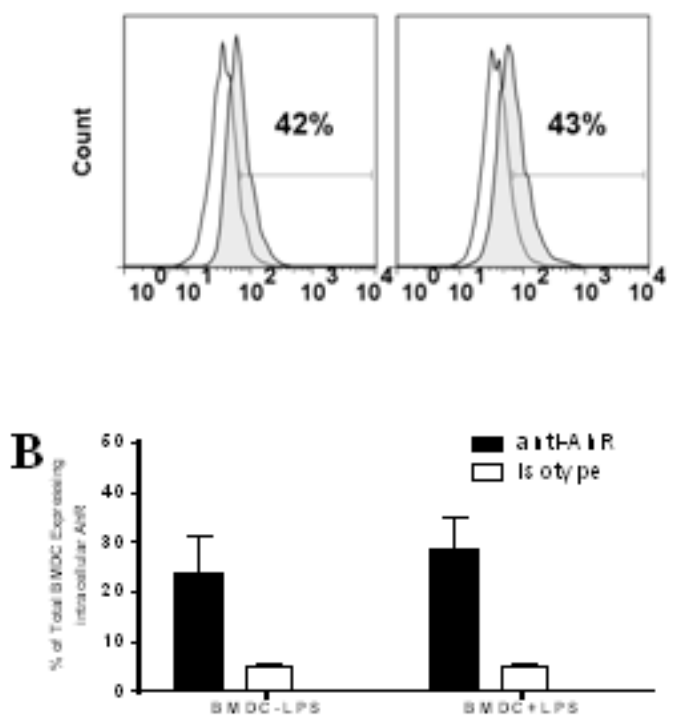

Figure 1. Immature and Mature BMDC Express Intracellular AhR.

BMDC were harvested on day 6, stimulated with or without $200 \mathrm{ng} / \mathrm{ml}$ LPS overnight and the following day cells were stained for surface MHC-II (maturation was confirmed by staining some samples with anti-CD11c, anti-CD86 or anti-CD40 or anti-MHC-II) and then intracellular AhR, while control samples were stained for surface MHC-II and isotype control antibody. (A) FACS plot showing the percentage of intracellular AhR-positive immature BMDC (minus LPS) BMDC (filled histogram) compared to control (outline histogram). (B) FACS plot showing the percentage of intracellular AhR-positive mature (plus LPS) BMDC (filled histogram) compared to control (outline histogram). (C) Mean percentages of AhR-positive cells in either immature (minus LPS) or mature BMDC (+LPS). Data are from 2 experiments $(n=4)$, samples were treated in triplicates, and mean values were plotted and significant differences were determined by ANOVA.

A

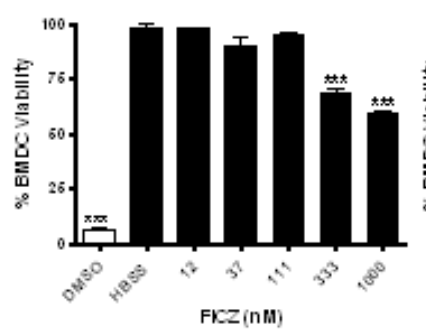

C
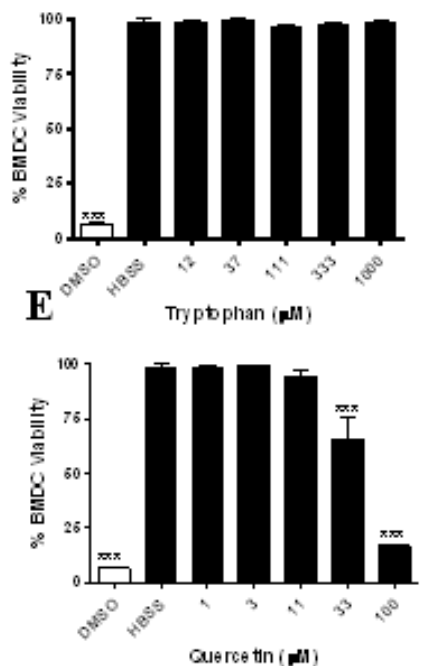

Figure 2. High Concentrations of Curcumin and Quercetin Suppress $B M D C$ Viability.

BMDC were harvested on day 6 , stimulated with different AhR-ligands, or HBSS alone (positive control for viability) or $5 \%$ DMSO (negative control for viability), and incubated overnight. The following day, cell viability was determined by addition of WST-1 reagent. The percent viability was calculated by normalization with $100 \%$ viable HBSS buffer control. (A) FICZ. (B) I3C. (C) Tryptophan. (D) Curcumin. (E) Quercetin. Data are from 2 experiments and all samples were treated in triplicates. Significantly reduced viability compared to HBSS control is indicated, *** $\mathrm{p}<0.001$. 
Representative data from 2 experiment $(n=4)$ are shown, mean value were plotted and significant differences were determined by ANOVA.

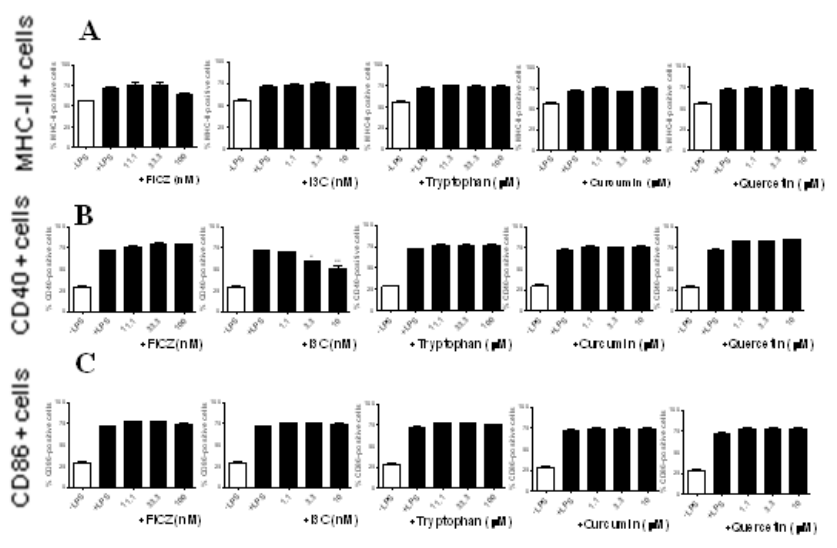

Figure 3. AhR-Ligands have no Significant Effect on Mature BMDC Surface Marker Expression.

BMDC were harvested on day 6 , and stimulated overnight with or without $200 \mathrm{ng} / \mathrm{ml}$ LPS and doses of different AhR-ligands: FICZ, I3C, tryptophan, curcumin and quercetin. The following day cells were stained for CD11c-positive BMDC and different maturation markers. (A) MHC class II expression, gated on CD11c-positive cells. (B) CD40 expression, gated on CD11c-positive cells. (C) CD86 expression, gated on CD11c-positive cells. No significant differences were observed between LPS + AhRligand-treated samples compared to LPS treatment alone, except the suppressive effect of I3C on CD40 expression by BMDC at high doses. Significantly reduced CD40 expression compared to LPS treatment alone was observed, ${ }^{*} \mathrm{p}<0.05,{ }^{*} \mathrm{p}<0.01$, respectively. Representative data from 2 experiment $(\mathrm{n}=4)$ are shown, mean values were plotted and significant differences were determined by ANOVA.

A

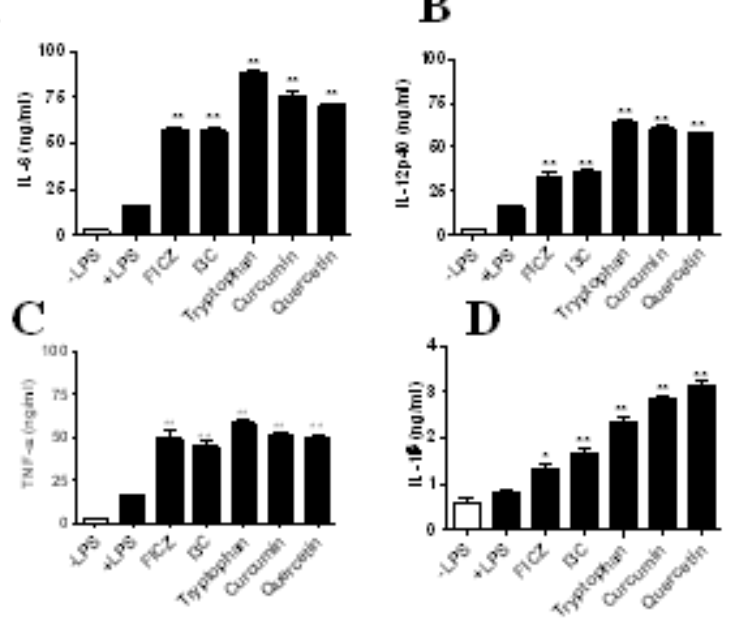

Figure 4. AhR-Ligands Increase the Secretion of Pro-inflammatory Cytokines by LPS-mMatured BMDC.

BMDC were harvested on day 6 , stimulated overnight with or without $200 \mathrm{ng} / \mathrm{ml}$ LPS and the following doses of
AhR-ligands: FICZ (100nM), I3C (100nM), tryptophan $(100 \mu \mathrm{M})$, curcumin $(10 \mu \mathrm{M})$ and quercetin $(10 \mu \mathrm{m})$. (A) LPS-treated-BMDC significantly increased the secretion of IL-6 as compared with immature-BMDC (-LPS), (** $\mathrm{p}<0.01)$. All AhR-ligands significantly induced the secretion of IL-6, as compared to BMDC treated with LPS alone $(* * p$ $<0.01)$. (B) LPS-treated-BMDC significantly increased the secretion of IL-12p40 as compared with immature-BMDC (-LPS), $(* * \mathrm{p}<0.01)$. All AhR-ligands significantly enhanced the secretion of IL-12p40, as compared to BMDC treated with LPS alone $(* * p<0.01)$. (C) LPS-treated-BMDC significantly increased the secretion of TNF- $\alpha$ as compared with immature-BMDC (-LPS), $\left({ }^{* *} \mathrm{p}<0.01\right)$. All AhR-ligands significantly increased the secretion of TNF- $\alpha$, as compared to BMDC treated with LPS alone, $(* * p<0.01)$. (D) LPS-treated-BMDC significantly increased the secretion of IL-1 $\beta$ as compared with immature-BMDC (-LPS), $(* * p<0.01)$. All AhR-ligands significantly induced the secretion of IL- $1 \beta$ as compared to control, $\left({ }^{*} p<0.05\right.$, $* * \mathrm{p}<0.01)$. Data are from 4 experiments $(\mathrm{n}=4)$ are shown, samples were treated in duplicates, mean values were plotted and significant differences were determined by ANOVA.

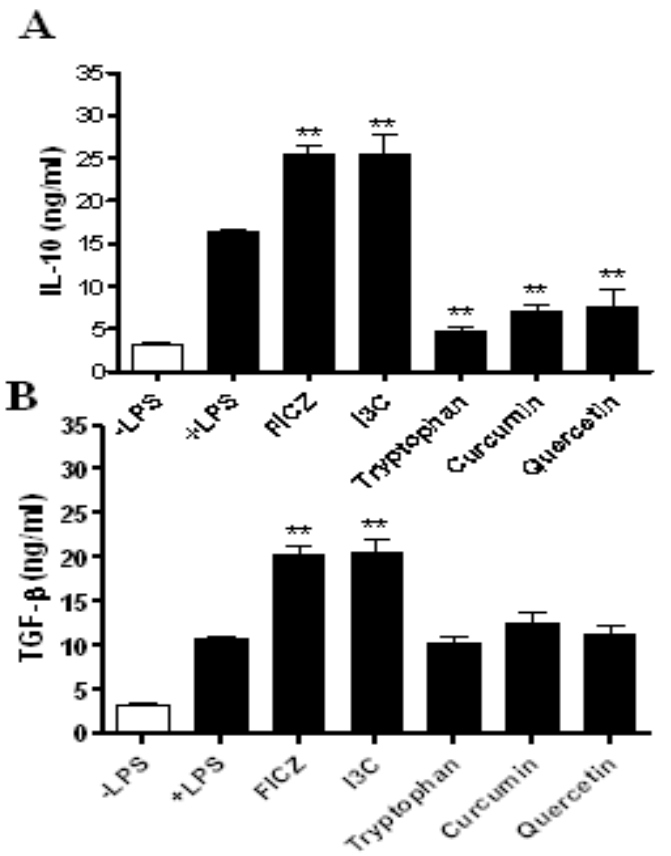

Figure 5. FICZ and I3C Enhance the Secretion of Anti-inflammatory Cytokines by LPS-Matured BMDC.

BMDC were harvested on day 6, stimulated overnight with or without $200 \mathrm{ng} / \mathrm{ml}$ LPS and the following doses of AhR-ligands: FICZ (100nM), I3C (100nM), tryptophan $(100 \mu \mathrm{M})$, curcumin $(10 \mu \mathrm{M})$, and quercetin $(10 \mu \mathrm{m})$. (A) LPS-treated-BMDC significantly increased the secretion of IL-10 as compared with immature-BMDC (-LPS), (** $\mathrm{p}<0.01)$. FICZ and I3C significantly increased the secretion of IL-10 by BMDC compared to LPS-treatment alone $(* * p<0.01)$. In contrast, tryptophan, curcumin and quercetion significantly decreased the secretion of IL-10 by BMDC as compared to LPS-treatment alone $(* * p<0.01)$. (B) 
LPS-treated-BMDC significantly increased the secretion of TGF- $\beta$ as compared with immature-BMDC (-LPS), $(* * p<0.01)$. FICZ and I3C significantly increased the secretion of TGF- $\beta$ by BMDC as compared to BMDC treated with LPS alone $(* * \mathrm{p}<0.01)$. Data are from 4 experiments $(\mathrm{n}=4)$, samples were treated in duplicates, mean values were plotted and significant differences were determined by ANOVA.

A
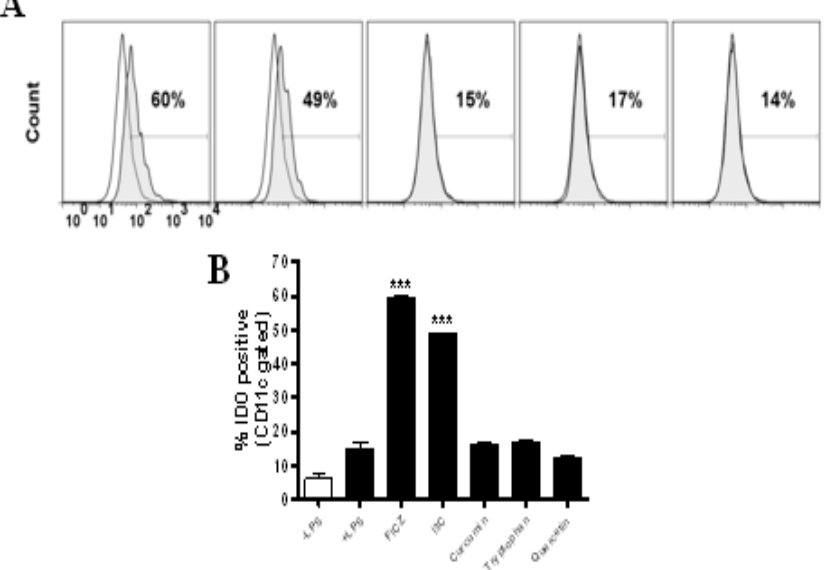

Figure 6. FICZ and I3C Significantly Enhance the Expression of Intracellular IDO in BMDC.

BMDC were harvested on day 6 , and stimulated overnight with or without $200 \mathrm{ng} / \mathrm{ml}$ LPS and the following doses or AhR-ligands: FICZ (100nM), I3C (100nM), tryptophan $(100 \mu \mathrm{M})$, curcumin $(10 \mu \mathrm{M})$ and quercetin $(10 \mu \mathrm{m})$. The following day, cells were stained for CD11c and then intracellular IDO expression. (A) Histograms showing percentage of IDO-positive cells (gated on CD11c-positive cells) after different AhR-ligand treatments. The outline curve illustrates the IDO staining of BMDC-treated with LPS alone, and the filled overlay shows the IDO staining of BMDC additionally treated with the indicated AhR-ligand. (B) Mean percent IDO-positive cells, gated on CD11c-positive matured BMDC. LPS-treated-BMDC significantly increased the expression of IDO as compared with immature-BMDC (minus LPS), $(* * p<0.01)$. Both FICZ and I3C significantly enhanced the expression of intracellular IDO in BMDC $(* * * \mathrm{p}<0.001)$. In contrast, tryptophan, curcumin and quercetion have no significant effect on the expression of IDO by BMDC as compared to LPS-treatment alone. Data are from 2 experiments $(n=4)$, samples were treated in duplicates, mean values were plotted, and significant differences were determined by ANOVA.

\section{Acknowledgements}

This study was supported by a New Investigator Award from the Medical Research Council (UK) and a Biotechnology and Biological Sciences Research Council (BBSRC) Project Grant (to D.G.M). H.A.A was supported by a PhD Studentship from the Government of Kuwait.

\section{References}

[1] Esser, C., A. Rannug, and B. Stockinger, The aryl hydrocarbon receptor in immunity. Trends Immunol, 2009. 30(9): p. 447-54.

[2] Cua, D.J. and C.M. Tato, Innate IL-17-producing cells: the sentinels of the immune system. Nat Rev Immunol, 2010. 10(7): p. 479-89.

[3] Komura, K., et al., Aryl hydrocarbon receptor/dioxin receptor in human monocytes and macrophages. Mol Cell Biochem, 2001. 226(1-2): p. 107-18.

[4] Frericks, M., et al., Transcriptional signatures of immune cells in aryl hydrocarbon receptor (AHR)-proficient and AHR-deficient mice. Biol Chem, 2006. 387(9): p. 1219-26.

[5] Denison, M.S. and S.R. Nagy, Activation of the aryl hydrocarbon receptor by structurally diverse exogenous and endogenous chemicals. Annu Rev Pharmacol Toxicol, 2003. 43: p. 309-34.

[6] Schecter, A., et al., Dioxins: an overview. Environ Res, 2006. 101(3): p. 419-28.

[7] Walker, L.S. and A.K. Abbas, The enemy within: keeping self-reactive $\mathrm{T}$ cells at bay in the periphery. Nat Rev Immunol, 2002. 2(1): p. 11-9.

[8] Sakaguchi, S., et al., Regulatory $\mathrm{T}$ cells and immune tolerance. Cell, 2008. 133(5): p. 775-87.

[9] Wan, Y.Y. and R.A. Flavell, How diverse--CD4 effector T cells and their functions. $J$ Mol Cell Biol, 2009. 1(1): p. 20-36.

[10] Murphy, K.M. and B. Stockinger, Effector T cell plasticity: flexibility in the face of changing circumstances. Nat Immunol, 2010. 11(8): p. 674-80.

[11] Vlad, G., R. Cortesini, and N. Suciu-Foca, License to heal: bidirectional interaction of antigen-specific regulatory $\mathrm{T}$ cells and tolerogenic APC. J Immunol, 2005. 174(10): p. 5907-14.

[12] Coombes, J.L. and F. Powrie, Dendritic cells in intestinal immune regulation. Nat Rev Immunol, 2008. 8(6): p. 435-46.

[13] Mellor, A.L. and D.H. Munn, IDO expression by dendritic cells: tolerance and tryptophan catabolism. Nat Rev Immunol, 2004. 4(10): p. 762-74.

[14] Mezrich, J.D., et al., An interaction between kynurenine and the aryl hydrocarbon receptor can generate regulatory $\mathrm{T}$ cells. J Immunol, 2010. 185(6): p. 3190-8.

[15] Nguyen, N.T., et al., Aryl hydrocarbon receptor negatively regulates dendritic cell immunogenicity via a kynurenine-dependent mechanism. Proc Natl Acad Sci U S A, 2010. 107(46): p. 19961-6.

[16] Vogel, C.F., et al., Aryl hydrocarbon receptor signaling mediates expression of indoleamine 2, 3-dioxygenase. Biochem Biophys Res Commun, 2008. 375(3): p. 331-5.

[17] Bankoti, J., et al., Functional and phenotypic effects of AhR activation in inflammatory dendritic cells. Toxicol Appl Pharmacol, 2010. 246(1-2): p. 18-28. 
[18] Simones, T. and D.M. Shepherd, Consequences of AhR activation in steady-state dendritic cells. Toxicol Sci, 2011. 119(2): p. 293-307.

[19] Apetoh, L., et al., The aryl hydrocarbon receptor interacts with c-Maf to promote the differentiation of type 1 regulatory $\mathrm{T}$ cells induced by IL-27. Nat Immunol, 2010. 11(9): p. 854-61.

[20] Gandhi, R., et al., Activation of the aryl hydrocarbon receptor induces human type 1 regulatory T cell-like and Foxp3 (+) regulatory T cells. Nat Immunol, 2010. 11(9): p. 846-53.

[21] Bankoti, J., et al., Effects of TCDD on the fate of naive dendritic cells. Toxicol Sci, 2010. 115(2): p. 422-34.

[22] Veldhoen, M., et al., The aryl hydrocarbon receptor links TH17-cell-mediated autoimmunity to environmental toxins. Nature, 2008. 453(7191): p. 106-9.

[23] Quintana, F.J., et al., Control of T(reg) and T(H)17 cell differentiation by the aryl hydrocarbon receptor. Nature, 2008. 453(7191): p. 65-71.

[24] Veldhoen, M., et al., Natural agonists for aryl hydrocarbon receptor in culture medium is essential for optimal differentiation of Th17 T cells. J Exp Med, 2009. 206(1): p. 43-9.

[25] Lutz, M.B., et al., An advanced culture method for generating large quantities of highly pure dendritic cells from mouse bone marrow. J Immunol Methods, 1999. 223(1): p. 77-92.

[26] Ciolino, H.P., et al., Effect of curcumin on the aryl hydrocarbon receptor and cytochrome P450 1A1 in MCF-7 human breast carcinoma cells. Biochem Pharmacol, 1998. 56(2): p. 197-206.

[27] Ciolino, H.P., P.J. Daschner, and G.C. Yeh, Dietary flavonols quercetin and kaempferol are ligands of the aryl hydrocarbon receptor that affect CYP1A1 transcription differentially. Biochem J, 1999. 340 ( Pt 3): p. 715-22.

[28] Bjeldanes, L.F., et al., Aromatic hydrocarbon responsiveness -receptor agonists generated from indole - 3-carbinol in vitro and in vivo: comparisons with 2, 3, 7, 8-tetrachlorodibenzo-p-dioxin. Proc Natl Acad Sci U S A, 1991. 88(21): p. 9543-7.

[29] Kerkvliet, N.I., Recent advances in understanding the mechanisms of TCDD immunotoxicity. Int Immunopharmacol, 2002. 2(2-3): p. 277-91.

[30] Hilkens, C.M., J.D. Isaacs, and A.W. Thomson, Development of dendritic cell-based immunotherapy for autoimmunity. Int Rev Immunol, 2010. 29(2): p. 156-83.

[31] Bright, J.J., Curcumin and autoimmune disease. Adv Exp Med Biol, 2007. 595: p. 425-51.

[32] Cong, Y., et al., Curcumin induces the tolerogenic dendritic cell that promotes differentiation of intestine-protective regulatory T cells. Eur J Immunol, 2009. 39(11): p. 3134-46.

[33] Kim, G.Y., et al., Curcumin inhibits immunostimulatory function of dendritic cells: MAPKs and translocation of NF-kappa B as potential targets. J Immunol, 2005. 174(12): p 8116-24.

[34] Jeong, Y.I., et al., Curcumin suppresses the induction of indoleamine 2,3-dioxygenase by blocking the Janus-activated kinase-protein kinase Cdelta-STAT1 signaling pathway in interferon- gamma- stimulated murine dendritic cells. J Biol Chem, 2009. 284(6): p. 3700-8.

[35] Jung, I.D., et al., COX-2 and PGE2 signaling is essential for the regulation of IDO expression by curcumin in murine bone marrow-derived dendritic cells. Int Immunopharmacol, 2010. 10(7): p. $760-8$.

[36] Huang, R.Y., et al., Immunosuppressive effect of quercetin on dendritic cell activation and function. J Immunol, 2011. 184(12): p. 6815-21.

[37] Miller, J.I., Modulation of Dendritic Cells and Autoimmunity by Apoptotic and Necrotic Cells, in Disease Department. 2011, The University of Manchester: London. p. 187.

[38] de Waard, W.J., et al., Gene expression profiling in Caco-2 human colon cells exposed to TCDD, benzo [a] pyrene, and natural $\mathrm{Ah}$ receptor agonists from cruciferous vegetables and citrus fruits. Toxicol In Vitro, 2008. 22(2): p. 396-410.

[39] Rinaldi, A.L., et al., Curcumin activates the aryl hydrocarbon receptor yet significantly inhibits (-) benzo (a) pyrene7R-trans- 7, 8-dihydrodiol bioactivation in oral squamous cell carcinoma cells and oral mucosa. Cancer Res, 2002. 62(19): p. 5451-6.

[40] Ashida, H., et al., Flavones and flavonols at dietary levels inhibit a transformation of aryl hydrocarbon receptor induced by dioxin. FEBS Lett, 2000. 476(3): p. 213-7.

[41] Zal, T., et al., Mechanisms of tolerance induction in major histocompatibility complex class II-restricted T cells Specific for a blood-borne self-antigen. J Exp Med, 1994. 180 (8): p. 2089-99. 